$>$ La question de l'alimentation des personnels hospitaliers n'a retenu l'attention que tardivement. Pourtant, la nourriture est un miroir fidèle dans lequel se reflètent les savoirs, les pouvoirs, les valeurs et les imaginaires d'une institution. C'est en 1905, que la réforme faite par l'Administration générale de l'Assistance Publique de Paris se préoccupe, pour la première fois, spécifiquement, de la nourriture des personnels hospitaliers. C'est un changement dans les pratiques, fondées jusque-là sur un principe d'égalité du traitement alimentaire de l'ensemble des hospitaliers, issu probablement du cadre monastique de l'hôpital des origines. L'instauration du système en vigueur aujourd'hui se fait progressivement dans les années 1930. Désormais, les personnels paient leur repas à l'administration qui en établit le compte sur la base d'une carte de réfectoire. Au terme de cette évolution, rien n'est plus pareil dans l'hôpital sur le terrain des relations hiérarchiques entre l'administration et ses personnels. L'ère des selfs et des plateaux-repas s'est ouverte, transformant le consommateur en un individu autonome, actif et participatif, dans une institution convertie au grand jeu de la démocratie d'entreprise. <

\section{Regards sur I'alimentation des personnels hospitaliers}

\section{Anne Nardin}

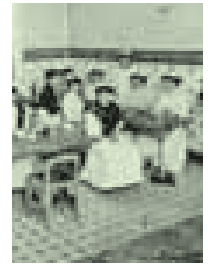

«Depuis que je dirige l'Administration de l'Assistance Publique, je n'ai pas eu de souci plus constant que celui de l'amélioration matérielle et morale du personnel hospitalier [...]. Un nouveau régime alimentaire, plus substantiel, plus varié [...] était mis en expérimentation dès les derniers mois de 1903 dans quelques établissements, et vient d'être étendu sans exception à tous nos hôpitaux et hospices ${ }^{1} \gg$.

En proclamant ses intentions de réformer l'alimentation des personnels hospitaliers, Gustave Mesureur, directeur, depuis 1902, de l'Administration générale de l'Assistance Publique de Paris, semblait rompre une tradition immuable. Pour la première fois, sans doute, une réforme de l'alimentation hospitalière concerne spécifiquement les personnels. Cette distinction introduit une rupture dans les pratiques hospitalières, fondées jusque-là sur un principe d'égalité du traitement alimentaire de l'ensemble des populations: administrés, patients et personnels.

Ce constat ne surprend guère: on sait à quel point, dans le passé, l'hôpital a représenté un milieu clos dont la logique saisissait tous ceux qui en franchissaient le seuil. En revanche, dans un contexte où la fonction de l'alimentation relève officiellement du projet thérapeutique (depuis la fin du XVIII ${ }^{e}$ siècle), cette proximité intrigue, en tant qu'elle est susceptible de mettre en cause la finalité médicale du régime des malades. Cette ambiguïté constitue l'un des paradoxes de l'alimentation hospitalière, comme prise entre deux projets dont

${ }^{1}$ Gustave Mesureur. Circulaire du $1^{\text {er }}$ décembre 1905. Archives de l'AP-HP, Registre des arrêtés et circulaires. 
les statuts et les enjeux respectifs s'opposent en partie: nourrir (l'intendance) ou soigner (le médical).

Le cas particulier de l'Assistance Publique de Paris est examiné ici, sur une période couvrant le $x \mid x^{e}$ siècle et la première moitié du $x x^{e}$ siècle.

\section{Un cadre unique pour tous}

\section{De la fonctionnalité avant toute chose?}

La tendance qui consiste à confondre, dans une même approche, l'alimentation des malades et celle des personnels correspond, à première vue, à une conception pragmatique et fonctionnelle de l'hôpital, qui à cet égard se présente d'abord comme une collectivité. Les malades et les personnels y constituent longtemps des populations stables et résidentes. Que l'on se place du point de vue de la cuisine, de celui de l'économat ou de la direction, une conception globale et quantitative s'impose. Le premier règlement relatif au régime alimentaire, publié par le Conseil Général des Hospices de Paris en 1806, exprime cette vision d'ensemble en identifiant toutes les populations concernées par ce règlement ${ }^{2}$ : les «employés et gens de service»; les «pensionnaires et indigents»; enfin, le «régime des infirmeries des Hospices» destiné aux malades. Est ainsi posé ce que le nouveau règlement de 1841 appellera les «trois classes de consommateurs», principe qui demeurera la référence jusqu'à la première moitié du $x x^{\mathrm{e}}$ siècle.

Pourtant, et bien qu'elle paraisse imparable, cette logique fonctionnelle et comptable n'épuise pas les raisons de ce constant parallélisme, dans les textes, entre malades et personnels. Deux autres explications sont à prendre en compte, qui tiennent à l'ordre symbolique des représentations.

\section{Une communauté de vie et de destin}

C'est probablement dans le cadre monastique de l'hôpital des origines que s'enracine cette proximité entre patients et personnels. On peut reconnaître dans l'infirmerie des monastères $d u v^{e}$ et $v l^{e}$ siècles la première ébauche de ce qui, trois siècles plus tard, deviendra l'hôpital ou hôtel-Dieu. L'accueil du pèlerin ou du nécessiteux y est vécu comme une ardente obligation, un précepte spirituel qui découle très directement de l'enseignement évangélique. Cette finalité définit une communauté de vie et de destin.

L'alimentation hospitalière en porte l'empreinte: le repas n'a d'autre but que de fortifier le corps pour le rendre apte au service de Dieu. II s'inscrit dans un cadre spirituel qui lui donne son sens. Manger ne doit pas séparer de Dieu, mais au contraire permettre de s'en rapprocher: non pas une occasion

\footnotetext{
${ }^{2}$ Régime alimentaire des Hospices Civils de Paris, 9 juillet 1806. Archives de l'Assistance publique-Hôpitaux de Paris.

${ }^{3}$ « Parce qu'il n'est pas bien que les maîtres (c'est-à-dire les malades) manquent de quelque chose, et que les serviteurs ( $c$ 'est-à-dire les religieux) vivent dans la magnificence ». Charte
} de l'Hôtel-Dieu de Troyes, citée dans Léon Lallemand ([1], tome 3, p. 190). de chute, mais une forme de louange et de reconnaissance pour le don renouvelé de la subsistance, un don qui ne saurait être accaparé $[1]^{3}$, mais au contraire qui appelle le partage, le don aux pauvres, c'est-à-dire à Dieu, («J'ai eu faim, et vous m'avez donné à manger»), dans un mouvement circulaire qui, seul, assurera en retour le renouvellement de la grâce divine. Rythmes, comportements et pratiques découlent de cet ordre religieux qui assigne une même attitude aux hospitaliers et aux malades - désirée ou consentie pour les uns, plus ou moins subie pour les autres - et oriente pour longtemps la culture hospitalière en matière d'alimentation.

\section{Le statut social du « petit personnel 》}

Si le choix d'une alimentation simple et austère correspond pour les religieuses à un choix de vie fondé sur un principe de souveraineté du spirituel sur le temporel, sa justification institutionnelle emprunte d'autres voies au XIX siècle, lorsque la gestion des hôpitaux parisiens échoit à la nouvelle administration du Conseil Général des Hospices. Certes, les religieuses sont encore présentes, jusqu'en 1908 pour la plupart, mais la convention qui les lie désormais à l'institution les range parmi les différentes catégories de son personnel. À leurs côtés, se trouve la masse des «gens de service, serviteurs et sous-serviteurs $\gg$, et, vers le milieu $d u x x^{e}$ siècle, les infirmiers et infirmières qui assureront, vers 1907 seulement, la relève des religieuses. À vrai dire, ce «petit personnel» est présent depuis longtemps dans les murs de l'hôpital, à proportion de la taille de l'établissement et du nombre de malades accueillis. Au $\mathrm{XIX}$ siècle, le recrutement continue de drainer des population issues de catégories sociales défavorisées, le «rebut des serviteurs incapables de pouvoir être conservés nulle part ailleurs» [2], pour lesquels l'hôpital représente un placement acceptable. L'héritage et la tradition orientent l'attitude de l'Assistance Publique au moment de l'émergence du nouveau corps laïque des infirmières. Cette attitude contraste avec le choix des écoles suisses et anglaises qui cherchent à recruter un personnel d'élite parmi les jeunes filles de bonne famille. L'admi-

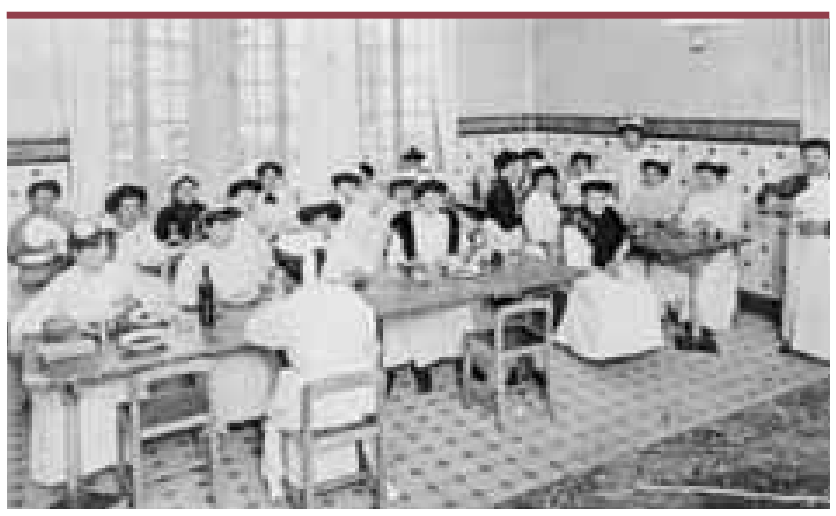

Un réfectoire d'infirmières à l'hospice de Brévannes, 1910 (๔) Archives de l'AP-HP). 
nistration prend donc clairement position en faveur d'un recrutement par le bas, revendiquant la possibilité d'une ascension sociale pour ses jeunes élèves de l'École de la Salpêtrière, issues de milieux populaires.

Ainsi, qu'il s'agisse du personnel soignant ou du personnel technique, les origines sociales des hospitaliers se confondent globalement, jusqu'au début du $x^{e}$ siècle, avec celles des malades et des administrés. Le jugement social dans lequel l'administration enferme, en quelque sorte, malades et personnels s'exprime, au niveau alimentaire, à travers de multiples indices. Ainsi, par exemple, les améliorations apportées au régime des malades concernent toujours dans le même temps le personnel : «J'ai décidé qu'à partir du $1^{\text {er }}$ septembre prochain les malades (adultes ou enfants), sauf contre-indication médicale, et les agents du personnel nourris, recevraient deux services de gâteaux frais par semaine au lieu d'un, de même que les pensionnaires des maisons de retraite et les administrés des hospices ${ }^{4} \gg$. En l'absence de telles mesures, les uns et les autres ne peuvent compter que sur la délivrance, par les médecins, de «bons de vivres supplémentaires» pour compléter un régime monotone et insuffisant. Tout se passe comme si, dans ce domaine de l'alimentation, la normalisation opérée par la médecine et les représentations à l'œuvre additionnaient leurs effets pour imposer un ordre unique.

\section{Exceptions faites...}

Il est cependant une catégorie de personnels qui, bien qu'elle apparaît parfois dans le corpus des textes règlementaires, semble échapper à cet ordre culinaire: ce sont les médecins. En effet, tout en ayant leur place dans l'un des réfectoires du personnel (organisation dont il sera question plus loin), les médecins édifient un système parallèle qui, en les séparant radicalement du reste des employés, leur permet, privilège exceptionnel, d'aménager un dispositif de restauration sur mesure. Ce système a pour cadre la salle de garde, où se rassemble, ponctuellement, l'ensemble de la communauté médicale.

Historiquement, les médecins ne s'assimileront jamais à la communauté hospitalière. D'un côté, leur foi dans le progrès, leur vision de la médecine et de sa finalité les placent en porte-à-faux, vis-à-vis des religieuses. De l'autre, il n'est pas question pour eux d'être inclus, c'est-à-dire enfermés, dans un rapport de symétrie entre malades et personnel, autrement dit d'adopter ce régime hospitalier, eux dont les origines sociales, dans la plupart des cas, et la culture leur ont appris un autre rapport à l'alimentation. Tout les pousse à refuser la morale religieuse et sociale qui modèle la cuisine hospitalière depuis des siècles. Il y va de leur identité. Peu à peu, un dispositif privilégié s'organise au sein de la salle de garde. L'admi-

${ }^{4}$ Circulaire du 23 août 1934. Archives de l'Assistance publique-Hôpitaux de Paris. Registre des arrêtés et circulaires.

${ }^{5}$ Pour plus de détails sur les traditions culinaires de la salle de garde, voir l'ouvrage de Patrick Josset [3]. nistration y fait régulièrement livrer les denrées nécessaires à la confection des repas et met à la disposition exclusive de ses occupants une cuisinière qui leur mitonne des plats d'une autre qualité que ceux servis aux malades et au personnel [3] ${ }^{5}$. Le bien-manger préserve ici ses droits et, au-delà, sert à affirmer très hautement une irréductible différence de statut et de condition.

\section{Hiérarchie, discipline et transgression}

La nature de l'alimentation marque ainsi la position de chacun. Mais ce sont aussi la structure hiérarchique et l'autorité de l'institution parisienne qui s'expriment fortement à travers les modalités du repas.

\section{Des réfectoires distincts}

La nouvelle administration du Conseil Général des Hospices, dans son «Règlement alimentaire des hospices civils» de 1806, distingue cinq réfectoires différents, séparant soigneusement les différentes catégories de personnel: $1^{\text {er }}$ réfectoire: agents de surveillance (c'est-à-dire les directeurs), économes, chapelains ; $2^{\mathrm{e}}$ réfectoire: élèves internes en médecine, chirurgiens, pharmaciens, employés de bureaux, instituteurs, piqueurs des bâtiments, chefs d'ateliers, surveillants(es) et reposants de même classe; $3^{e}$ réfectoire: sous-surveillants(es) et reposants de même classe ; $4^{\mathrm{e}}$ réfectoire: gens de service des deux sexes et reposants de même classe ; $5^{e}$ réfectoire : ouvriers, hommes de peine et cuisiniers.

Est-ce la conséquence d'un assouplissement des relations hiérarchiques ou la volonté de simplifier une gestion lourde et compliquée, toujours est-il qu'il ne reste plus que trois réfectoires dans le nouveau règlement de 1841 , puis deux dans celui de 1867: le premier réservé au personnel gradé, le second au personnel non gradé. Les archives ne permettent pas de situer à quelle date le dispositif tombe en désuétude. On sait seulement, par la réforme de Gustave Mesureur, que les différences de régime et de quantité qui distinguaient les réfectoires sont supprimées en 1905.

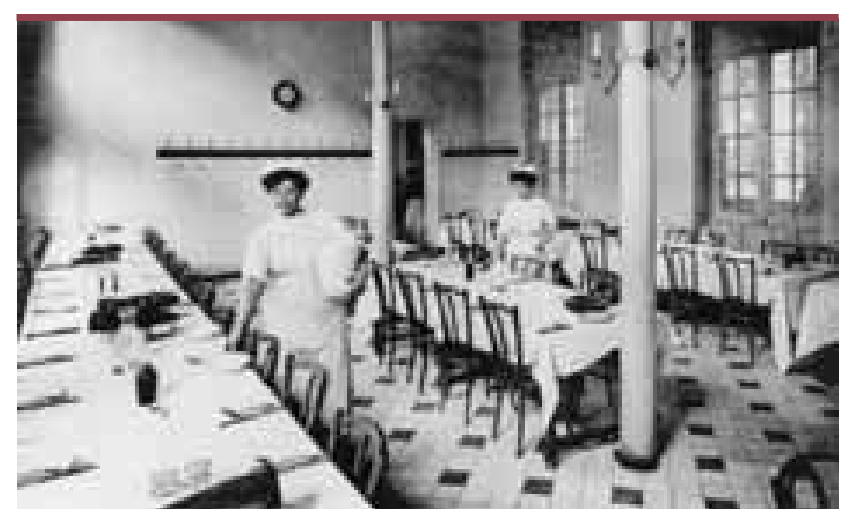

Réfectoire des infirmiers, Hôpital Tenon, Paris, 1910 (ㄷ) Archives de I'AP-HP). 


\section{À travers les mailles du filet}

Le cadre disciplinaire qui organise ce qui a trait à la nourriture traduit aussi la fréquence des tentatives de transgression de la part des personnels. Depuis le Moyen Âge, les archives en témoignent, ce ne sont qu'abus et trafics permanents que l'institution tente, vainement semble-t-il, de contenir et de réprimer. Ces abus et ces trafics expriment d'une part un profond besoin d'évasion vis-à-vis d'un ordre culinaire austère, d'autre part le souci de se procurer un peu de liquidités ; ils disent enfin la tentation permanente que représente l'abondance des vivres en circulation, abondance qui fait, même à tort, assimiler l'hôpital à une véritable poule aux œufs d'or. La trop grande distorsion entre cette apparente profusion et ce qui est servi dans l'assiette, semble justifier un jeu clandestin de compensation, contre lequel, bien sûr, l'institution se défend en multipliant contrôles et règlements.

\section{Écarts et différences}

Tous les personnels ne sont donc pas logés à la même enseigne. $\mathrm{Si}$ le menu quotidien est le même pour tous, préparé dans un lieu unique avec les mêmes produits, il existe cependant des variations qui signent l'appartenance hiérarchique des bénéficiaires. Parallèlement, des différences subsistent, parfois subtiles, qui maintiennent une séparation entre la population des malades et celle des bien-portants. D'autres, enfin, se construisent au cours $\mathrm{du} \mathrm{xx}^{\mathrm{e}}$ siècle, signes d'une mutation lente mais profonde des conditions de travail et du statut des personnels hospitaliers.

\section{Des malades toujours prioritaires}

Certaines situations affectent différemment la population des malades et celle des bien-portants, lorsque des prescriptions morales ou des contraintes économiques modifient le cours du quotidien. Laissant de côté le cas des «jours maigres» et autres carêmes de l'Ancien Régime, nous ne retiendrons ici qu'un exemple, celui des restrictions en temps de guerre. Ainsi, lors du Dernier Conflit mondial, les premières restrictions s'imposent au personnel dès le 13 octobre 1939 et concernent la viande de boucherie. De nouvelles mesures entrent en vigueur au début de l'année 1940 pour le personnel et les assistés, et portent sur le pain et les pâtisseries, les malades étant alors toujours épargnés. Mais les restrictions de sucre, décidées en juin 1940, concernent cette fois les malades, dont les tisanes sont préparées sans sucre. Seuls le personnel et les administrés se voient servir de la soupe au petit déjeuner en remplacement du café sucré. Ce n'est qu'en juillet 1940 que la suppression du plat de légumes s'applique indistinctement à toutes les populations hospitalières ${ }^{6}$. La situation ne fera que se dégrader au cours des mois suivants.

${ }^{6}$ Voir circulaires du 28 octobre 1939, du 26 mars 1940, du 26 juin 1940 et du 8 juillet 1940 Archives de l'Assistance publique-Hôpitaux de Paris. Registre des arrêtés et circulaires.

\section{Le salaire de la peine}

À côté du critère de l'état de santé, qui fait des malades une population généralement reconnue prioritaire, à l'inverse, ceux de l'effort à fournir et de la pénibilité du travail valent quelques améliorations au régime des personnels. Certaines catégories bénéficient d'allocations spécifiques. Les gardiens d'amphithéâtres, les agents chargés du triage du linge sale et ceux chargés du blanchissage des articles de pansement bénéficient, depuis 1908 pour les premiers, d'un supplément de « $50 \mathrm{cl}$ de thé additionné de rhum, à raison de $6 \mathrm{cl}$ de rhum pour $1 \mid$ de thé $\gg^{7}$. Ce petit privilège disparaît en 1959 , lorsque l'administration découvre les inconvénients de cette délivrance, beaucoup plus grands pour la santé des agents que l'illusoire bénéfice qu'ils peuvent en tirer à titre «prophylactique ${ }^{8}$.

\section{Ni pauvres, ni malades}

Quelque chose de cet ancien monde fermé sur ses traditions et enfermé dans ses représentations se lézarde au début du $x x^{e}$ siècle. La circulaire de Mesureur nous paraît en constituer le premier signe, dans la mesure où elle se préoccupe spécifiquement, pour la première fois, de la nourriture des personnels. II ne s'agit d'ailleurs pas d'une action isolée: elle s'inscrit au contraire dans un éventail de mesures comme l'augmentation générale des salaires, le relèvement des taux de pension, la réglementation plus libérale des sorties hebdomadaires et de la durée de la journée de travail. Elle n'est sans doute pas séparable du mouvement de réflexion qui agite l'institution au sujet de la formation, imminente à cette date, d'un nouveau personnel infirmier destiné à remplacer les religieuses. L'heure est à la revalorisation de ce personnel «servant», dans lequel sont rangées les infirmières jusqu'en 1903. Pour autant, l'ensemble des réformes décidées par Gustave Mesureur demeure de portée modeste.

L'instauration du système en vigueur aujourd'hui se fait progressivement dans les années 1930. Désormais, les personnels paient leur repas à l'administration qui en établit le compte sur la base d'une carte de réfectoire. Pour anodin que puisse paraître ce changement, il induit une relation différente du consommateur à la nourriture consommée, mais aussi de l'employé à l'employeur. Parallèlement à l'évolution de la législation du travail, le changement opéré dans le deuxième tiers de notre siècle suit en fin de compte l'émancipation progressive des personnels vis-à-vis d'un cadre en décalage avec l'évolution de la société, des mœurs et des aspirations individuelles. Ce changement devient indispensable lorsque le personnel, mieux rémunéré, choisit de se loger à l'extérieur de l'hôpital, autrement dit décide de se ménager un

${ }^{7}$ Circulaires du 12 février, du 20 février et du 31 décembre 1908, du 16 juin 1939 et du 20 février 1947. Archives de l'Assistance publique-Hôpitaux de Paris. Registre des arrêtés et circulaires, AP-HP.

${ }^{8}$ Circulaire du 7 décembre 1959. Archives de l'Assistance publique-Hôpitaux de Paris. Registre des arrêtés et circulaires. 
espace et un temps de vie privés. Dès lors, prendre ses repas à l'hôpital ne représente plus guère qu'une commodité. Payer son repas signifie qu'il appartient à celui qui le consomme ; c'est aussi symboliquement s'appartenir à soi-même. Ces nouveaux principes se mettent en place vers $1935^{\circ}$ et se généralisent dès $1942^{10}$. L'indexation du prix du repas sur le niveau de salaire des employés est instaurée en $1951^{11}$.

Ainsi, au terme de cinquante années d'évolution, rien n'est plus pareil dans l'hôpital sur le terrain des relations hiérarchiques entre l'administration et ses personnels. L'employé ne vient plus au réfectoire à l'heure dite, le couvert dans sa poche. L'ère des selfs et des plateaux-repas s'est ouverte, transformant le consommateur en un individu autonome, actif et participatif, dans une institution convertie au grand jeu de la démocratie d'entreprise. $\diamond$

${ }_{9}^{9}$ Circulaire du 6 février 1935. Institution d'une carte de repas pour les agents nourris (à ce moment, la carte ne sert qu'à comptabiliser les présences du personnel aux réfectoires). Archives de l'Assistance publique-Hôpitaux de Paris. Registre des arrêtés et circulaires.

${ }^{10}$ Note du 7 décembre 1942. Tickets-repas pour paiement des repas pris dans les réfectoires par le personnel hospitalier. Archives de l'Assistance publique-Hôpitaux de Paris. Registre des arrêtés et circulaires.

${ }^{11}$ Note du 19 février 1951. Archives de l'Assistance publique-Hôpitaux de Paris. Registre des arrêtés et circulaires.

\section{SUMMARY}

Considerations regarding the food of the hospital staff

The question concerning the food of the hospital staff has drawn attention only tardily. However, food is a faithful mirror in which the knowledge, the capacities, the values and the imaginary ones of an institution are reflected. It is in 1905 that the reform made by the «Administration générale de l'Assistance Publique de Paris » is specifically concerned, for the first time, by the food of the hospital staff. It is a change in the practices, founded until then on the principle of equality in food treatment, resulting probably from the monastic form of the hospital in the earliest times. The introduction of the system into force today was done gradually in the years 1930 . From now on, hospital staff pay their meal to the administration which establishes the account of it on the basis of a refectory card. At the end of this evolution, nothing is similar any more in the hospital on the ground of the hierarchical relations between the administration and its staff. The era of self-service restaurants has open, transforming the consumer into an autonomous active and participative individual, in an institution converted into the play of democraty company. $\diamond$

\section{RÉFÉRENCES}

1. Lallemand L. Histoire de la charité, 4 tomes, 5 vol. Paris : Picard, 1902-1912.

2. Prevost M. Paris-Guide. Paris : Librairie Internationale, 1867.

3. Josset P. La salle de garde. Histoire et signification des rituels des salles de garde de médecine, chirurgie et pharmacie du Moyen-Âge à nos jours. Paris : Éditions Le Léopard d'Or, 1997.

\section{TIRÉS À PART}

A. Nardin

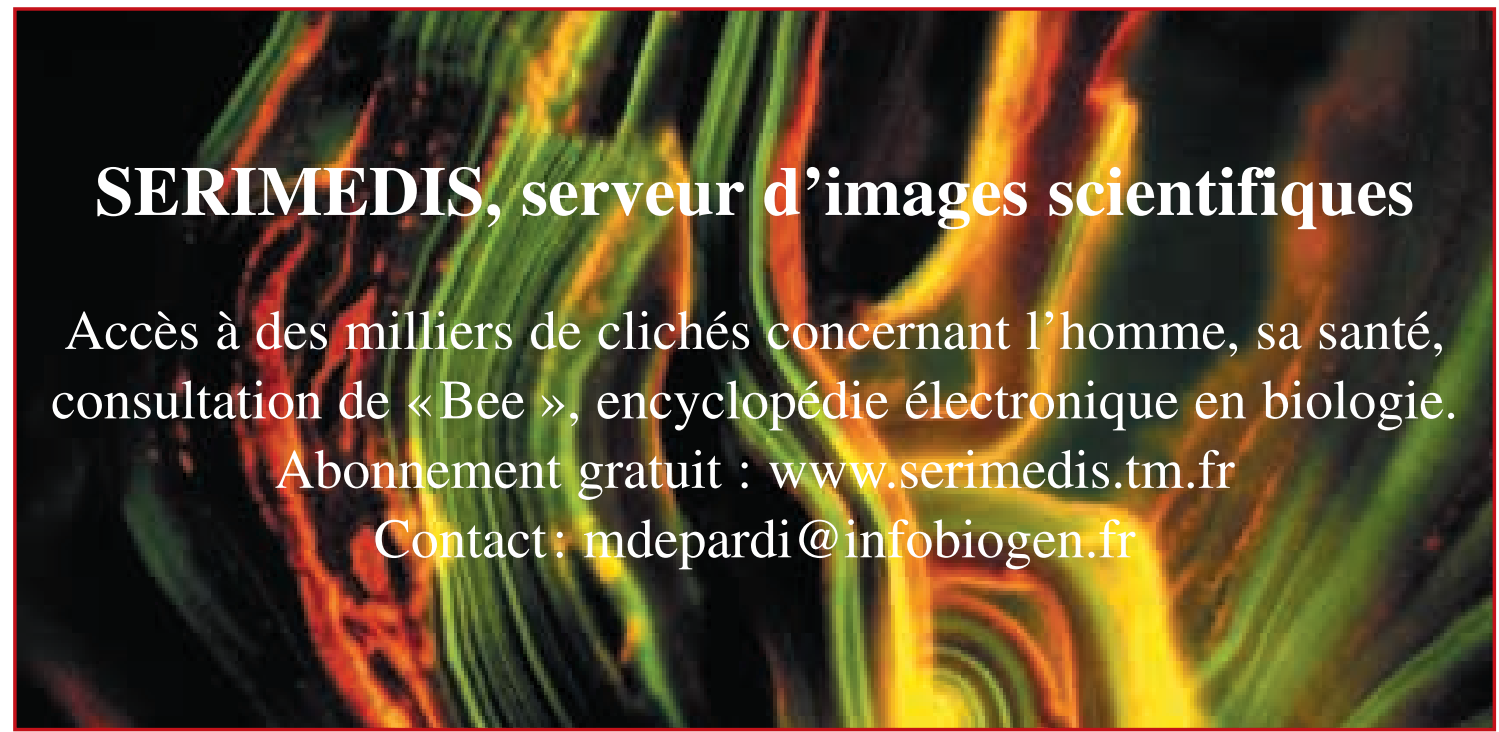

